UFIFT-QG-07-05

\title{
Scalar Field Equations from Quantum Gravity during Inflation
}

\author{
E. O. Kahya ${ }^{\dagger}$ and R. P. Woodard ${ }^{\ddagger}$ \\ Department of Physics \\ University of Florida \\ Gainesville, FL 32611
}

\begin{abstract}
We exploit a previous computation of the self-mass-squared from quantum gravity to include quantum corrections to the scalar evolution equation. The plane wave mode functions are shown to receive no significant one loop corrections at late times. This result probably applies as well to the inflaton of scalar-driven inflation. If so, there is no significant correction to the $\varphi \varphi$ correlator that plays a crucial role in computations of the power spectrum.
\end{abstract}

PACS numbers: 04.60.-m, 04.62.+v, 98.80.Cq, 98.80.Qc

† e-mail: emre@phys.ufl.edu

‡ e-mail: woodard@phys.ufl.edu 


\section{Introduction}

Massless, minimally coupled scalars and gravitons are unique in possessing zero mass without classical conformal invariance. Simple arguments based on the energy-time uncertainty principle suggest that quantum effects from these particles should be vastly enhanced during primordial inflation [1]. The lowest order effect is that inflation rips long wavelength virtual quanta of these types out of the vacuum. That is the origin of the observed scalar perturbations [2] and of the potentially observable tensor perturbations [3].

Higher order effects derive from the interactions of the sea of long wavelength virtual scalars and gravitons, both with themselves and with other particles. Loop corrections to the primordial power spectra are enhanced by logarithms of the ratio of the scale factor to its value at first horizon crossing [4, 5]. For any mode whose spatial variation we can resolve today these secular enhancements cannot overcome the minuscule loop counting parameter of $G H^{2} \lesssim 10^{-12}$. Exceptions have been suggested [6, 7, 8]. However, much larger loop corrections (to other things) can be obtained either from interactions with larger loop counting parameters, or by studying things we would perceive as spatially constant such as the vacuum energy or particle masses.

Explicit computations have been made on de Sitter background in five different models which involve either scalars or gravitons:

1. For a massless, minimally coupled scalar with a quartic self-interaction - the two loop expectation value of the stress tensor [9] and the two loop corrections to the scalar mode function [10, 11].

2. For a massless, minimally coupled scalar which is Yukawa-coupled to a massless fermion - the one loop corrections to the fermion [12, 13] and scalar [14] mode functions, and the two loop vertex function [15].

3. For massless, minimally coupled scalar quantum electrodynamics the one loop correction to the photon [16, 17] and scalar [18] mode functions, the two loop corrections to the coincident scalar [19] and field strength [20] bilinears and to the stress tensor [20].

4. For general relativity - the one loop self-energy [21] and the expectation value of the graviton field at one [22, 23, 24] and two loops [25].

5. For general relativity coupled to a massless fermion - the one loop corrected fermion mode functions [26]. 
The leading secular effects of the first three models agree precisely with the stochastic formalism of Starobinskiu [27, 28, 29], and the series of these leading effects at all orders can be resummed [30, 15, 31] to give nonperturbative predictions. No stochastic results are available for the last two models.

It is natural to extend these studies by combining general relativity with a massless, minimally coupled scalar to probe the effects of gravitons on scalars. Such an investigation has great phenomenological interest because the inflaton potential of scalar-driven inflation is so flat that inflaton mode functions are effectively those of a massless, minimally coupled scalar. Indeed, it is standard to compute the power spectrum of scalar perturbations by setting the scalar-scalar correlator to its value for a massless, minimally coupled scalar on de Sitter background [32, 33]. The subject of quantum gravitational corrections to the scalar effective potential has a long history [34, 35] but we will here look at all one loop corrections to the linearized, effective scalar field equation, including corrections to the derivative terms and also the fully nonlocal corrections. This is what one must do to fix the scalar field strength in addition to its mass. It is worth noting that one loop quantum gravitational corrections induce a time dependent field strength renormalization for massless fermions which eventually becomes nonperturbatively large [26].

Our result is that the scalar mode functions experience no significant corrections at one loop. We prove this by solving the linearized, effective scalar field equation. Section 2 gives a brief review of our previous one loop computation of the scalar self-mass-squared [36], which represents the quantum correction to the linearized effective field equation. In section 3

we actually solve the equation in the relevant regime of late times. Our conclusions comprise section 4 .

\section{Our Previous Calculation}

The linearized effective scalar field equation consists of the classical term minus the scalar self-mass-squared $M^{2}\left(x ; x^{\prime}\right)$ integrated against the scalar,

$$
\partial_{\mu}\left(\sqrt{-g} g^{\mu \nu} \partial_{\nu} \varphi(x)\right)-\int d^{4} x^{\prime} M^{2}\left(x ; x^{\prime}\right) \varphi\left(x^{\prime}\right)=0 .
$$


We computed $M^{2}\left(x ; x^{\prime}\right)$ at one loop order using the bare Lagrangian of Einstein + Scalar,

$$
\mathcal{L} \equiv-\frac{1}{2} \partial_{\mu} \varphi \partial_{\mu} \varphi g^{\mu \nu} \sqrt{-g}+\frac{1}{16 \pi G}(R-(D-2) \Lambda) \sqrt{-g} .
$$

Here $\Lambda \equiv(D-1) H^{2}$ is the cosmological constant and $G$ is Newton's constant. We worked on the open conformal coordinate patch of $D$-dimensional de Sitter space, with the graviton field $h_{\mu \nu}$ defined as,

$$
g_{\mu \nu}(\eta, \vec{x}) \equiv a^{2}(\eta)\left(\eta_{\mu \nu}+\kappa h_{\mu \nu}(\eta, \vec{x}) \quad \text { where } \quad a(\eta) \equiv-\frac{1}{H \eta} .\right.
$$

Here $\eta_{\mu \nu}$ is the Minkowski metric and $\kappa^{2} \equiv 16 \pi G$ is the loop counting parameter of quantum gravity. The graviton propagator was computed by adding a non-de Sitter invariant gauge fixing term [37, 38],

$$
\begin{aligned}
\mathcal{L}_{\mathrm{GF}} & \equiv-\frac{1}{2} \sqrt{-g} g^{\mu \nu} F_{\mu} F_{\nu}, \\
F_{\mu} & \equiv \eta^{\rho \sigma}\left(h_{\mu \rho, \sigma}-\frac{1}{2} h_{\rho \sigma, \mu}+(D-2) a H h_{\mu \rho} \delta_{\sigma}^{0}\right) .
\end{aligned}
$$

The computation of $M^{2}\left(x ; x^{\prime}\right)$ was made using dimensional regularization. We subtracted off the one loop divergences using three dimension six counterterms,

$$
\begin{aligned}
& \Delta \mathcal{L}=\frac{1}{2} \alpha_{1} \kappa^{2} \varphi_{; \mu \nu} \varphi_{; \rho \sigma} g^{\mu \nu} g^{\rho \sigma} \sqrt{-g} \\
& \quad-\frac{\alpha_{2} \kappa^{2} R}{2 D(D-1)} \varphi_{, \mu} \varphi_{, \nu} g^{\mu \nu} \sqrt{-g}-\frac{\alpha_{3} \kappa^{2} R}{2 D(D-1)} \varphi_{, i} \varphi_{, j} g^{i j} \sqrt{-g}
\end{aligned}
$$

In these expressions a comma denotes ordinary differentiation while a semicolon stands for the covariant derivative. The noninvariant counterterm proportional to $\alpha_{3}$ was made necessary by our non-de Sitter invariant gauge fixing function (5). Because gravity is not perturbatively renormalizable, only the divergent parts of the coefficients $\alpha_{i}$ are fixed,

$$
\begin{aligned}
& \alpha_{1}=0+\Delta \alpha_{1} \\
& \alpha_{2}=\frac{H^{D-4}}{(4 \pi)^{\frac{D}{2}}}\left\{\frac{61}{3}\right\}+\Delta \alpha_{2} \\
& \alpha_{3}=\frac{H^{D-4}}{(4 \pi)^{\frac{D}{2}}}\left\{\frac{4}{D-4}-\frac{58}{3}-2 \gamma\right\}+\Delta \alpha_{3} .
\end{aligned}
$$




\begin{tabular}{|c|c|}
\hline External Operator & Coefficient of $\frac{\kappa^{2} H^{4}}{(4 \pi)^{4}}$ \\
\hline$\left(a a^{\prime}\right)^{4} \square^{3} / H^{2}$ & $-\frac{\ln x}{3 x}$ \\
\hline$\left(a a^{\prime}\right)^{4} \square^{2}$ & $\frac{26 \ln x}{3 x}+\frac{38}{3 x}-6 \ln 2$ \\
& \\
\hline$\left(a a^{\prime}\right)^{4} H^{2} \square$ & $-\frac{6 \ln x}{x}+\frac{4}{x}-4 \ln x$ \\
\hline$\left(a a^{\prime}\right)^{4} H^{4}$ & $\frac{4 \ln x}{x}+\frac{18}{x}-108 \ln x-120$ \\
\hline$\left(a a^{\prime}\right)^{3}\left(a^{2}+a^{\prime 2}\right) \square^{3} / H^{2}$ & $\frac{\ln x}{6 x}$ \\
\hline$\left(a a^{\prime}\right)^{3}\left(a^{2}+a^{\prime 2}\right) \square^{2}$ & $-\frac{\ln x}{3 x}+\frac{1}{6 x}$ \\
\hline$\left(a a^{\prime}\right)^{3}\left(a^{2}+a^{\prime 2}\right) H^{2} \square$ & $-\frac{2 \ln x}{3 x}+\frac{5}{x}-18 \ln x$ \\
\hline$\left(a a^{\prime}\right)^{3}\left(a^{2}+a^{\prime 2}\right) H^{4}$ & $\frac{4 \ln x}{3 x}-\frac{32}{3 x}-54$ \\
\hline$\left(a a^{\prime}\right)^{3} H^{2} \nabla^{2}$ & $-\frac{2 \ln x}{3 x}-\frac{16}{x}+84 \ln x-48 x \ln x+96 x$ \\
\hline$\left(a a^{\prime}\right)^{3} \nabla^{2} \square$ & $\frac{\ln x}{3 x}$ \\
\hline$\left(a a^{\prime}\right)^{2}\left(a^{2}+a^{\prime 2}\right) H^{2} \nabla^{2}$ & $-\frac{7 \ln x}{3 x}+\frac{11}{2 x}-12 \ln x+48 x \ln x+12 x$ \\
\hline$\left(a a^{\prime}\right)^{2}\left(a^{2}+a^{2}\right) \nabla^{2} \square$ & $-\frac{17 \ln x}{6 x}-\frac{49}{6 x}+4 \ln { }^{2} x+10 \ln x+12 x \ln x$ \\
\hline$\left(a a^{\prime}\right)^{2} \nabla^{4}$ & $\frac{10}{3} \ln x-24 x \ln x+24 x^{2} \ln x-36 x^{2}$ \\
\hline
\end{tabular}

Table 1: Nonlocal Contributions to $-i M^{2}\left(x ; x^{\prime}\right)$. Here $x \equiv \frac{y}{4}$ and $y\left(x ; x^{\prime}\right)$ is defined in equation (12).

The finite parts $\Delta \alpha_{i}$ are arbitrary.

After renormalization and taking the unregulated limit $(D=4)$, our result is [36],

$$
-i M^{2}\left(x ; x^{\prime}\right)=i \kappa^{2} a^{4}\left(\Delta \alpha_{1} \square^{2}+\Delta \alpha_{2} \square+\Delta \alpha_{3} \frac{\nabla^{2}}{a^{2}}\right) \delta^{4}\left(x-x^{\prime}\right)+(\text { Table 1 }) .
$$

The Laplacian $\nabla^{2}$ and the d'Alembertian $\square$ are,

$$
\nabla^{2} \equiv \partial_{i} \partial_{i} \quad \text { and } \quad \square \equiv \frac{1}{a^{4}} \partial^{\mu}\left(a^{2} \partial_{\mu}\right)=-\frac{1}{a^{2}} \partial_{0}^{2}-\frac{2}{a} H \partial_{0}+\frac{1}{a^{2}} \nabla^{2} .
$$

We should also define the de Sitter invariant length function that appears in Table 1,

$$
y\left(x ; x^{\prime}\right) \equiv a(\eta) a\left(\eta^{\prime}\right)\left\{\left\|\vec{x}-\vec{x}^{\prime}\right\|^{2}-\left(\left|\eta-\eta^{\prime}\right|-i \delta\right)^{2}\right\} .
$$




\section{Effective Mode Equation}

This is the heart of the paper. We begin by clarifying what is meant by the effective mode equation, then we explain the restricted sense in which we solve it. Finally we work out the contributions from the local counterterms in (10) and from the nonlocal terms of Table 1 .

\subsection{The Schwinger-Keldysh Formalism}

We seek to find plane wave mode solutions to "the effective field equations." Although the quoted phrase is common parlance, it is nonetheless ambiguous because there are different sorts of effective field equations whose solutions mean different things in terms of the unique canonical operator formalism. Introductory courses in quantum field theory typically concern the in-out effective field equations. The plane wave mode solutions of these equations give in-out matrix elements of commutators of the full field with a tree order creation operator of the in vacuum,

$$
\Phi_{\text {io }}(x ; \vec{k})=\left\langle\Omega_{\text {out }}\left|\left[\varphi(x), \alpha_{\text {in }}^{\dagger}(\vec{k})\right]\right| \Omega_{\text {in }}\right\rangle .
$$

This quantity is of great interest for flat space scattering problems but it has little relevance to cosmology where there may be an initial singularity and where particle production precludes the in vacuum from evolving to the out vacuum.

The more interesting cosmological experiment is to release the universe from a prepared state at finite time and let it evolve as it will. The mode solutions of interest to this experiment are the expectation values of commutators of the full field with the tree order creation operator of the initial vacuum,

$$
\Phi(x ; \vec{k})=\left\langle\Omega\left|\left[\varphi(x), \alpha^{\dagger}(\vec{k})\right]\right| \Omega\right\rangle .
$$

The effective field equation that $\Phi(x ; \vec{k})$ obeys is given by the SchwingerKeldysh formalism [26, 18]. This is a covariant covariant extension of Feynman diagrams which produces true expectation values instead of in-out matrix elements [39, 40, 41, 42, Because there are excellent reviews on this subject [43, 44, 45, 46], we will confine ourselves to explaining how to use the formalism.

The chief difference between the Schwinger-Keldysh and in-out formalisms is that the endpoints of particle lines have a \pm polarity. Therefore, every 
propagator $i \Delta\left(x ; x^{\prime}\right)$ of the in-out formalism gives rise to four SchwingerKeldysh propagators: $i \Delta_{++}\left(x ; x^{\prime}\right), i \Delta_{+_{-}}\left(x ; x^{\prime}\right), i \Delta_{-+}\left(x ; x^{\prime}\right)$ and $i \Delta_{--}\left(x ; x^{\prime}\right)$. Each of these propagators can be obtained by making simple changes to the Feynman propagator. For our model, the Feynman propagators of the scalar and graviton happen to depend upon the length function $y\left(x ; x^{\prime}\right)$ defined in expression (12), and also upon the two scale factors. The four polarities derive from making the following substitutions for $y\left(x ; x^{\prime}\right)$ :

$$
\begin{array}{ll}
i \Delta_{++}\left(x ; x^{\prime}\right): & y \rightarrow y_{++}\left(x ; x^{\prime}\right) \equiv a(\eta) a\left(\eta^{\prime}\right)\left[\left\|\vec{x}-\vec{x}^{\prime}\right\|^{2}-\left(\left|\eta-\eta^{\prime}\right|-i \delta\right)^{2}\right], \\
i \Delta_{+-}\left(x ; x^{\prime}\right): & y \rightarrow y_{+-}\left(x ; x^{\prime}\right) \equiv a(\eta) a\left(\eta^{\prime}\right)\left[\left\|\vec{x}-\vec{x}^{\prime}\right\|^{2}-\left(\eta-\eta^{\prime}+i \delta\right)^{2}\right], \\
i \Delta_{-+}\left(x ; x^{\prime}\right): & y \rightarrow y_{-+}\left(x ; x^{\prime}\right) \equiv a(\eta) a\left(\eta^{\prime}\right)\left[\left\|\vec{x}-\vec{x}^{\prime}\right\|^{2}-\left(\eta-\eta^{\prime}-i \delta\right)^{2}\right], \\
i \Delta_{--}\left(x ; x^{\prime}\right): & y \rightarrow y_{--}\left(x ; x^{\prime}\right) \equiv a(\eta) a\left(\eta^{\prime}\right)\left[\left\|\vec{x}-\vec{x}^{\prime}\right\|^{2}-\left(\left|\eta-\eta^{\prime}\right|+i \delta\right)^{2}\right] .
\end{array}
$$

Vertices in the Schwinger-Keldysh formalism either have all + lines or all lines. The + vertex is identical to that of the in-out formalism, whereas the - vertex is its conjugate.

Because any external line can be either + or - , each $\mathrm{N}$-point one particle irreducible (1PI) function of the in-out formalism gives rise to $2^{N} 1 \mathrm{PI}$ functions in the Schwinger-Keldysh formalism. The Schwinger-Keldysh effective action is the generating functional of these 1PI functions. We can express it in terms of fields $\varphi_{+}$, to access the + lines, and $\varphi_{-}$, to access the - lines,

$$
\begin{aligned}
& \Gamma\left[\varphi_{+}, \varphi_{-}\right]=S\left[\varphi_{+}\right]-S\left[\varphi_{-}\right]-\frac{1}{2} \int d^{4} x \int d^{4} x^{\prime} \\
& \qquad\left\{\begin{array}{c}
\varphi_{+}(x) M_{++}^{2}\left(x ; x^{\prime}\right) \varphi_{+}\left(x^{\prime}\right)+\varphi_{+}(x) M_{+-}^{2}\left(x ; x^{\prime}\right) \varphi_{-}\left(x^{\prime}\right) \\
+\varphi_{-}(x) M_{-+}^{2}\left(x ; x^{\prime}\right) \varphi_{+}\left(x^{\prime}\right)+\varphi_{-}(x) M_{--}^{2}\left(x ; x^{\prime}\right) \varphi_{-}\left(x^{\prime}\right)
\end{array}\right\}+O\left(\varphi_{ \pm}^{3}\right),
\end{aligned}
$$

where $S[\varphi]$ is the classical scalar action.

At the order we are working, $-i M_{++}^{2}\left(x ; x^{\prime}\right)$ is the same as the in-out selfmass-squared. We can therefore read it off from (10). We get $-i M_{+-}^{2}\left(x ; x^{\prime}\right)$ by dropping the delta function terms, reversing the sign and replacing $y\left(x ; x^{\prime}\right)$ by $y_{+-}\left(x ; x^{\prime}\right)$ in Table 1. The other two 1PI 2-point functions derive from conjugating these two,

$$
-i M_{--}^{2}\left(x ; x^{\prime}\right)=\left(-i M_{++}^{2}\left(x ; x^{\prime}\right)\right)^{*},-i M_{-+}^{2}\left(x ; x^{\prime}\right)=\left(-i M_{+-}^{2}\left(x ; x^{\prime}\right)\right)^{*} .
$$


To get the Schwinger-Keldysh effective field equations one varies the action with respect to the field of either polarity, then sets the two polarities equal to $\Phi(x)$. At linearized level this gives,

$$
a^{4} \square \Phi(x)-\int_{\eta_{i}}^{0} d \eta^{\prime} \int d^{3} x^{\prime}\left\{M_{++}^{2}\left(x ; x^{\prime}\right)+M_{+-}^{2}\left(x ; x^{\prime}\right)\right\} \Phi\left(x^{\prime}\right)=0 .
$$

Here $\eta_{i}=-1 / H$ is the initial (conformal) time at which the universe is released in free Bunch-Davies vacuum. One can see from relations (15) and (16), and from the extra conjugated vertex in $M_{+-}\left(x ; x^{\prime}\right)$, that the bracketed term vanishes for $\eta^{\prime}>\eta$. The fact that $y_{+-}\left(x ; x^{\prime}\right)$ is the complex conjugate of $y_{++}\left(x ; x^{\prime}\right)$ for $\eta^{\prime}<\eta$ means that the bracketed term is real. One also sees that it must involve the imaginary part of at least one propagator, which means the only net effect comes from points $x^{\prime \mu}$ on or inside the past lightcone of $x^{\mu}$. Hence the Schwinger-Keldysh effective field equations are real and causal, unlike those of the in-out formalism.

\subsection{Restrictions on Our Solution}

Two limitations on our knowledge impose important restrictions on the sense in which we can solve (21):

1. We only know the scalar self-mass-squared at one loop order; and

2. We took the initial state to be free, Bunch-Davies vacuum.

The first limitation means we must solve (21) perturbatively. The full scalar self-mass-squared can be expanded in powers of the loop-counting parameter $\kappa^{2}=16 \pi G$,

$$
M_{++}^{2}\left(x ; x^{\prime}\right)+M_{+-}^{2}\left(x ; x^{\prime}\right)=\sum_{\ell=1}^{\infty} \kappa^{2 \ell} \mathcal{M}_{\ell}^{2}\left(x ; x^{\prime}\right) .
$$

A similar expansion applies for plane wave solutions to (21),

$$
\Phi(x ; \vec{k})=\sum_{\ell=0}^{\infty} \kappa^{2 \ell} \Phi_{\ell}(\eta, k) \times e^{i \vec{k} \cdot \vec{x}} .
$$

To make $\Phi(x ; \vec{k})$ agree with (14) we must normalize the tree order solution appropriately,

$$
\Phi_{0}(\eta, k)=u(\eta, k) \equiv \frac{H}{\sqrt{2 k^{3}}}\left(1-\frac{i k}{a H}\right) \exp \left[\frac{i k}{a H}\right] .
$$


The $\ell \geq 1$ solutions obey,

$$
a^{2}\left[\partial_{0}^{2}+2 H \partial_{0}+k^{2}\right] \Phi_{\ell}(\eta, k)=-\sum_{k=1}^{\ell} \int_{\eta_{i}}^{0} d \eta^{\prime} \int d^{3} x^{\prime} \mathcal{M}_{k}^{2}\left(x ; x^{\prime}\right) \Phi_{\ell-k}\left(\eta^{\prime}, k\right) e^{i \vec{k} \cdot\left(\vec{x}^{\prime}-\vec{x}\right)}
$$

We know only $\mathcal{M}_{1}^{2}\left(x ; x^{\prime}\right)$ so the sole correction we can compute is $\Phi_{1}(\eta, k)$.

The second limitation means it only makes sense to solve for $\Phi_{1}(\eta, k)$ at late times, i.e., as $\eta \rightarrow 0^{-}$. Interactions result in important corrections to free vacuum on a flat background and it is unthinkable that this does not happen as well for de Sitter background. In the Schwinger-Keldysh formalism these corrections would correspond to vertices on the initial value surface [22, 18]. In the in-out formalism the free vacuum is automatically corrected by time evolution. One can follow the progress of this in the Schwinger-Keldysh formalism by isolating terms that decay with increasing time after the release of the initial state. For example, the two loop expectation value of the stress tensor of a massless, minimally coupled scalar with a quartic self-interaction gives the following energy density and pressure [9],

$$
\begin{aligned}
& \rho=\frac{\lambda H^{4}}{(2 \pi)^{4}}\left\{\frac{1}{8} \ln ^{2}(a)+\frac{1}{18 a^{3}}-\frac{1}{8} \sum_{n=1}^{\infty} \frac{(n+2) a^{-n-1}}{(n+1)^{2}}\right\}+O\left(\lambda^{2}\right), \\
& p=\frac{\lambda H^{4}}{(2 \pi)^{4}}\left\{-\frac{1}{8} \ln ^{2}(a)-\frac{1}{12} \ln (a)-\frac{1}{24} \sum_{n=1}^{\infty} \frac{\left(n^{2}-4\right) a^{-n-1}}{(n+1)^{2}}\right\}+O\left(\lambda^{2}\right) .
\end{aligned}
$$

We suspect that the (separately conserved) terms which fall like powers of $1 / a$ can be absorbed into an order $\lambda$ correction of the initial state. On the other hand, the terms which grow like powers of $\ln (a)$ represent the effect of inflationary particle production (in this case, of scalars) pushing the field up its quartic potential.

Because we have not worked out the order $\kappa$ and $\kappa^{2}$ corrections to BunchDavies vacuum, we cannot trust corrections to $\Phi_{1}(\eta, k)$ that fall off at late times relative to the tree order solution $u(\eta, k)$,

$$
u(\eta, k)=\frac{H}{\sqrt{2 k^{3}}}\left\{1+\frac{k^{2}}{2 H^{2} a^{2}}+\frac{i k^{3}}{3 H^{3} a^{3}}+O\left(\frac{k^{4}}{H^{4} a^{4}}\right)\right\} .
$$

To understand what this means, it is best to convert equation (25) for $\Phi_{1}$ from conformal time $\eta$ to comoving time $t \equiv-\ln (-H \eta) / H$,

$$
\left[\frac{\partial^{2}}{\partial t^{2}}+3 H \frac{\partial}{\partial t}+\frac{k^{2}}{a^{2}}\right] \Phi_{1}=-\frac{1}{a^{4}} \int_{\eta_{i}}^{0} d \eta^{\prime} u\left(\eta^{\prime}, k\right) \int d^{3} x^{\prime} \mathcal{M}_{1}^{2}\left(x ; x^{\prime}\right) e^{i \vec{k} \cdot\left(\vec{x}^{\prime}-\vec{x}\right)} .
$$


At late times we can drop the factor of $k^{2} / a^{2}$ on the left hand side. Given a putative form for the late time behavior of the right hand side it is easy to infer the leading late time behavior of $\Phi_{1}$, for example,

$$
\begin{aligned}
& \text { r.h.s } \longrightarrow \ln (a) \Longrightarrow \Phi_{1} \longrightarrow \frac{\ln ^{2}(a)}{6 H^{2}}, \\
& \text { r.h.s } \longrightarrow 1 \Longrightarrow \Phi_{1} \longrightarrow \frac{\ln (a)}{3 H^{2}}, \\
& \text { r.h.s } \longrightarrow \frac{\ln (a)}{a} \Longrightarrow \Phi_{1} \longrightarrow \text { Constant }-\frac{\ln (a)}{2 H^{2} a}, \\
& \text { r.h.s } \longrightarrow \frac{1}{a} \Longrightarrow \Phi_{1} \longrightarrow \text { Constant }-\frac{1}{2 H^{2} a} .
\end{aligned}
$$

Because any constant contribution to $\Phi_{1}$ could be absorbed into a field strength renormalization, the only effects which can be distinguished from corrections to the initial state derive from contributions to the right hand side that fall off no faster than $1 / \ln (a)$. It turns out that inverse powers of $\ln (a)$ cannot occur, so the practical dividing line is between contributions to the right hand side which grow or approach a nonzero constant and those which fall off.

\subsection{Local Corrections}

Because the scalar d'Alembertian annihilates the tree order solution, only the third, noncovariant counterterm makes any contribution to (29),

$$
\begin{aligned}
& \frac{1}{a^{4}} \int d^{4} x^{\prime} a^{4}\left(\Delta \alpha_{1} \square^{2}+\Delta \alpha_{2} \square+\right.\left.\Delta \alpha_{3} \frac{\nabla^{2}}{a^{2}}\right) \delta^{4}\left(x-x^{\prime}\right) \\
& \times u\left(\eta^{\prime}, \vec{k}\right) e^{i \vec{k} \cdot\left(\vec{x}^{\prime}-\vec{x}\right)}=-\Delta \alpha_{3} \frac{k^{2}}{a^{2}} u(\eta, k) .
\end{aligned}
$$

This term rapidly redshifts to zero and we see from the preceding discussion that it cannot make a significant contribution to $\Phi_{1}$ at late times.

\subsection{Nonlocal Corrections}

Although Table 1 might seem to present a bewildering variety of nonlocal contributions to (29), a series of seven straightforward steps suffices to evaluate each one: 
1. Eliminate any factors of $1 / y$ using the identities,

$$
\begin{aligned}
\frac{4}{y} & =\frac{\square}{H^{2}}\left\{\ln \left(\frac{y}{4}\right)\right\}+3 \\
\frac{4}{y} \ln \left(\frac{y}{4}\right) & =\frac{\square}{H^{2}}\left\{\frac{1}{2} \ln ^{2}\left(\frac{y}{4}\right)-\ln \left(\frac{y}{4}\right)\right\}+3 \ln \left(\frac{y}{4}\right)-2 .
\end{aligned}
$$

2. Extract the factors of $\square$ and $\nabla^{2}$ from the integration over $x^{\prime \mu}$ using the identities,

$$
\begin{aligned}
& \frac{\square}{H^{2}} \longrightarrow-\left[a^{2} \frac{\partial^{2}}{\partial a^{2}}+4 a \frac{\partial}{\partial a}+\frac{k^{2}}{a^{2} H^{2}}\right] \\
& \nabla^{2} \longrightarrow-k^{2} .
\end{aligned}
$$

3. Combine the ++ and +- terms to extract a factor of $i$ and make causality manifest,

$$
\begin{aligned}
& \ln \left(\frac{y_{++}}{4}\right)-\ln \left(\frac{y_{+-}}{4}\right)=2 \pi i \theta(\Delta \eta-\Delta x) \\
& \ln ^{2}\left(\frac{y_{++}}{4}\right)-\ln ^{2}\left(\frac{y_{+-}}{4}\right)=4 \pi i \theta(\Delta \eta-\Delta x) \ln \left(\frac{1}{4} a a^{\prime} H^{2}\left(\Delta \eta^{2}-\Delta x^{2}\right)\right) .
\end{aligned}
$$

Here we define $a \equiv a(\eta), a^{\prime} \equiv a\left(\eta^{\prime}\right), \Delta \eta \equiv \eta-\eta^{\prime}$ and $\Delta x \equiv\left\|\vec{x}-\vec{x}^{\prime}\right\|$. Note that any positive powers of $y$ become,

$$
y_{+ \pm} \longrightarrow-\frac{1}{4} a a^{\prime} H^{2}\left(\Delta \eta^{2}-\Delta x^{2}\right) \text {. }
$$

4. Make the change of variables $\vec{r}=\vec{x}^{\prime}-\vec{x}$, perform the angular integrations and make the further change of variable $r=\Delta \eta \cdot z$,

$$
\begin{aligned}
\int d^{3} x^{\prime} \theta(\Delta \eta-\Delta x) F\left(\frac{1}{4} a a^{\prime} H^{2}\left(\Delta \eta^{2}-\Delta x^{2}\right)\right) e^{i \vec{k} \cdot\left(\vec{x}^{\prime}-\vec{x}\right)} \\
=4 \pi \theta(\Delta \eta) \int_{0}^{\Delta \eta} d r r^{2} F\left(\frac{1}{4} a a^{\prime} H^{2}\left(\Delta \eta^{2}-r^{2}\right)\right) \frac{\sin (k \Delta x)}{k \Delta x} \\
=4 \pi \theta(\Delta \eta) \Delta \eta^{3} \int_{0}^{1} d z z^{2} F\left(a a^{\prime}\left(\frac{1}{a^{\prime}}-\frac{1}{a}\right)^{2}\left(\frac{1-z^{2}}{4}\right)\right) \frac{\sin (k \Delta \eta z)}{k \Delta \eta z}
\end{aligned}
$$

5. Reduce the $z$ integration to a combination of elementary functions and sine and cosine integrals [14. 
6. Make the change of variables $a^{\prime}=-1 / H \eta^{\prime}$, expand the integrand and perform the integration over $a^{\prime}$.

7. Act any derivatives with respect to $a$.

Much of the labor involved in implementing these steps derives from the spacetime dependence of the zeroth order solution, $u\left(\eta^{\prime}, k\right) e^{i \vec{k} \cdot \vec{x}^{\prime}}$. For example, one can see from (43) that only elementary functions would result from the $z$ integration if the zeroth order solution were constant. In fact it is completely justified to make this simplification,

$$
\begin{aligned}
&-\frac{1}{a^{4}} \int_{\eta_{i}}^{0} d \eta^{\prime} \int d^{3} x^{\prime} \mathcal{M}_{1}^{2}\left(x ; x^{\prime}\right) u\left(\eta^{\prime}, k\right) e^{i \vec{k} \cdot\left(\vec{x}^{\prime}-\vec{x}\right)} \\
& \longrightarrow-\frac{u(0, k)}{a^{4}} \int_{\eta_{i}}^{0} d \eta^{\prime} \int d^{3} x^{\prime} \mathcal{M}_{1}^{2}\left(x ; x^{\prime}\right)
\end{aligned}
$$

To see why, note from expressions (28) and (43) that the deviation of the zeroth order solution from $u(0, k)$ introduces at least two factors of $1 / a^{\prime}$ or $1 / a$. Because the constant mode function can at best result in powers of $\ln (a)$, and because it makes no physical sense to retain contributions to (29) which fall off, we can immediately discard terms which acquire an extra factor of $1 / a$. Extra factors of $1 / a^{\prime}$ effectively restrict the integration to early times, which again causes the net result to fall off at late times.

One consequence of the simplification (44) is that we can neglect any contribution from Table 1 which contains a factor of $\nabla^{2}$. We therefore need only compute terms of the form,

$$
-u(0, k) \frac{i H^{8} a^{4-K}}{(4 \pi)^{4}}\left(\frac{\square}{H^{2}}\right)^{N} \int_{\eta_{i}}^{0} d \eta^{\prime} a^{\prime K} \int d^{3} x^{\prime}\left\{f\left(\frac{y_{++}}{4}\right)-f\left(\frac{y_{+-}}{4}\right)\right\},
$$

where the constant $K$ takes the values of 3,4 and 5 , and the functions $f(x)$ are $1 / x, \ln x / x, \ln x$ and $\ln ^{2} x$. The action of the d'Alembertian derives from setting $k=0$ in (37). The integrations for $K=3$ (given in Table 2) and $K=4$ (given in Table 3) were worked out in a previous paper [19]. Applying the same technique — which is just the 7-step procedure given above for $k=0$ - gives the results for $K=5$ in Table 4 .

It remains just to act the d'Alembertians and sum the results for each of the contributions from Table1. That is done in Table 5. Although individual contributions can grow as fast as $\ln ^{2}(a)$, all growing or even finite terms 


\begin{tabular}{|c|c|}
\hline$f(x)$ & $-\frac{i H^{4}}{16 \pi^{2}} \times \int d^{4} x^{\prime} a^{\prime 3}\left\{f\left(\frac{y_{++}}{4}\right)-f\left(\frac{y_{+-}}{4}\right)\right\}$ \\
\hline \hline$\frac{1}{x}$ & $-\frac{\ln (a)}{a}+\frac{1}{a}+O\left(\frac{1}{a^{2}}\right)$ \\
\hline$\frac{\ln (x)}{x}$ & $-\frac{\ln ^{2}(a)}{2 a}+\frac{2 \ln (a)}{a}-\frac{3}{a}+\frac{\pi^{2}}{3 a}+O\left(\frac{\ln (a)}{a^{2}}\right)$ \\
\hline $\ln (x)$ & $\frac{1}{6}-\frac{\ln (a)}{2 a}+\frac{1}{4 a}+O\left(\frac{1}{a^{2}}\right)$ \\
\hline $\ln ^{2}(x)$ & $\frac{1}{3} \ln (a)-\frac{11}{9}-\frac{\ln ^{2}(a)}{2 a}+\frac{2 \ln (a)}{a}-\frac{9}{4 a}+\frac{\pi^{2}}{3 a}+O\left(\frac{\ln (a)}{a^{2}}\right)$ \\
\hline \hline
\end{tabular}

Table 2: Integrals with $a^{\prime 3}$.

\begin{tabular}{|c|c|}
\hline$f(x)$ & $-\frac{i H^{4}}{16 \pi^{2}} \times \int d^{4} x^{\prime} a^{\prime 4}\left\{f\left(\frac{y_{++}}{4}\right)-f\left(\frac{y_{+-}}{4}\right)\right\}$ \\
\hline \hline$\frac{1}{x}$ & $-\frac{1}{2}+O\left(\frac{1}{a}\right)$ \\
\hline$\frac{\ln (x)}{x}$ & $\frac{3}{4}+O\left(\frac{1}{a}\right)$ \\
\hline $\ln (x)$ & $\frac{1}{6} \ln (a)-\frac{11}{36}+O\left(\frac{1}{a}\right)$ \\
\hline $\ln ^{2}(x)$ & $\frac{1}{6} \ln ^{2}(a)-\frac{8}{9} \ln (a)+\frac{7}{4}-\frac{\pi^{2}}{9}+O\left(\frac{\ln (a)}{a}\right)$ \\
\hline \hline
\end{tabular}

Table 3: Integrals with $a^{44}$.

\begin{tabular}{|c|c|}
\hline$f(x)$ & $-\frac{i H^{4}}{16 \pi^{2}} \times \int d^{4} x^{\prime} a^{\prime 5}\left\{f\left(\frac{y_{++}}{4}\right)-f\left(\frac{y_{+-}}{4}\right)\right\}$ \\
\hline \hline$\frac{1}{x}$ & $-\frac{1}{6} a+O\left(\frac{1}{a}\right)$ \\
\hline$\frac{\ln (x)}{x}$ & $\frac{17}{36} a+O\left(\frac{1}{a}\right)$ \\
\hline $\ln (x)$ & $\frac{1}{24} a-\frac{1}{6}+O\left(\frac{1}{a}\right)$ \\
\hline $\ln ^{2}(x)$ & $-\frac{13}{144} a-\frac{1}{3} \ln (a)+\frac{5}{9}+O\left(\frac{\ln (a)}{a}\right)$ \\
\hline \hline
\end{tabular}

Table 4: Integrals with $a^{\prime 5}$. 
cancel. It follows that the right hand side of (29) falls off at least as fast as $1 / a$ times powers of $\ln (a)$. Hence there are no significant corrections to the mode function at one loop order.

\section{Discussion}

We have solved the one loop-corrected, linearized effective field equation for a massless, minimally coupled scalar interacting with gravity during de Sitter inflation. Unlike previous analyses of the scalar effective potential [35], our technique uses the full self-mass-squared, including corrections to the derivative terms and nonlocal corrections. It should therefore be sensitive not only to the scalar's mass but also to its field strength. We find no significant corrections of any sort at one loop order.

Physically our result means that the sea of infrared gravitons produced by inflation has little effect on the scalar. Although our scalar is a spectator to $\Lambda$ driven inflation, typical scalar inflaton potentials are so flat that the inflaton is also unlikely to suffer significant one loop corrections from quantum gravity. If so, it is consistent to use the tree order scalar mode functions to compute the power spectrum of cosmological perturbations.

There are good reasons why the scalar should not acquire a mass from quantum gravity [35] but there seems to be no reason why its field strength cannot suffer a time dependent renormalization of the type experienced by massless fermions [26]. Weinberg's result for the power spectrum allows for $\ln (a)$ corrections [4, and they do arise from individual terms in Table 5. However, there is no net correction of this form at one loop.

In the end, our null result may not be as surprising as it seems. It is very simple to show that the scalar self-mass-squared vanishes at one loop order on a flat background [36]. It is also wrong to think of the scalar as weaker than the fermion; what is weaker is the fractional one loop correction. Although massless fermions experience a time-dependent field strength renormalization, this represents a $\ln (a)$ enhancement of tree order mode functions which

fall like $1 / a^{\frac{3}{2}}$. By contrast, the tree order scalar mode functions approach a nonzero constant. Indeed, it is the fact that derivatives of this constant vanish which makes the fractional correction of the $\kappa h \partial \varphi \partial \varphi$ coupling so small. The sea of infrared gravitons is present, but it can only couple, at this order, to the scalar's stress-energy, and the stress-energy of a single scalar redshifts to zero at late times. 


\begin{tabular}{|c|c|}
\hline External Operator $\times f(x)$ & Coefficient of $u(0, k) \times \frac{H^{4}}{16 \pi^{2}}$ \\
\hline$\left(a a^{\prime}\right)^{4} \square^{3} / H^{2} \times-\frac{\ln x}{3 x}$ & 0 \\
\hline$\left(a a^{\prime}\right)^{4} \square^{2} \times \frac{26 \ln x}{3 x}$ & 0 \\
\hline$\left(a a^{\prime}\right)^{4} \square^{2} \times \frac{38}{3 x}$ & 0 \\
\hline$\left(a a^{\prime}\right)^{4} \square^{2} \times-6 \ln { }^{2} x$ & -18 \\
\hline$\left(a a^{\prime}\right)^{4} \square^{2} \times-18 \ln x$ & 0 \\
\hline$\left(a a^{\prime}\right)^{4} H^{2} \square \times-\frac{6 \ln x}{x}$ & 0 \\
\hline$\left(a a^{\prime}\right)^{4} H^{2} \square \times \frac{4}{x}$ & 2 \\
\hline$\left(a a^{\prime}\right)^{4} H^{2} \square \times-4 \ln x$ & 3 \\
\hline$\left(a a^{\prime}\right)^{4} H^{4} \times \frac{4 \ln x}{x}$ & -9 \\
\hline$\left(a a^{\prime}\right)^{4} H^{4} \times \frac{18}{x}$ & $33-18 \ln (a)$ \\
\hline$\left(a a^{\prime}\right)^{4} H^{4} \times-108 \ln x$ & $\left.\frac{-325+12 \pi^{2}}{27}\right)+\frac{14}{3} \ln (a)-\frac{2}{3} \ln ^{2}(a)$ \\
\hline$\left(a a^{\prime}\right)^{3}\left(a^{2}+a^{\prime 2}\right) \square^{3} / H^{2} \times \frac{\ln x}{6 x}$ & $\left(\frac{85-12 \pi^{2}}{27}\right)-4 \ln (a)+\frac{2}{3} \ln ^{2}(a)$ \\
\hline$\left(a a^{\prime}\right)^{3}\left(a^{2}+a^{\prime 2}\right) \square^{2} \times-\frac{\ln x}{3 x}$ & $\frac{8}{9}-\frac{2}{3} \ln (a)$ \\
\hline$\left(a a^{\prime}\right)^{3}\left(a^{2}+a^{\prime 2}\right) \square^{2} \times \frac{1}{6 x}$ & $\frac{55}{3}-10 \ln (a)$ \\
\hline$\left(a a^{\prime}\right)^{3}\left(a^{2}+a^{\prime 2}\right) H^{2} \square \times-\frac{2 \ln x}{3 x}$ & $\left(\frac{160-12 \pi^{2}}{27}\right)-\frac{10}{3} \ln (a)+\frac{2}{3} \ln ^{2}(a)$ \\
\hline$\left(a a^{\prime}\right)^{3}\left(a^{2}+a^{\prime 2}\right) H^{2} \square \times \frac{5}{x}$ & $-\frac{80}{9}+\frac{32}{3} \ln (a)$ \\
\hline$\left(a a^{\prime}\right)^{3}\left(a^{2}+a^{\prime 2}\right) H^{2} \square \times-18 \ln x$ & 0 \\
\hline$\left(a a^{\prime}\right)^{3}\left(a^{2}+a^{\prime 2}\right) H^{4} \times \frac{4 \ln x}{3 x}$ & $\left(\frac{-91+12 \pi^{2}}{27}\right)+\frac{8}{3} \ln (a)-\frac{2}{3} \ln ^{2}(a)$ \\
\hline$\left(a a^{\prime}\right)^{3}\left(a^{2}+a^{\prime 2}\right) H^{4} \times-\frac{32}{3 x}$ & - \\
\hline \hline $\operatorname{Total}$ & 0 \\
\hline \hline
\end{tabular}

Table 5: $\quad-\frac{u(0, k)}{a^{4}} \frac{i H^{8}}{(4 \pi)^{4}} \int d^{4} x^{\prime}$ (Ext. Operator) $\times\left\{f\left(\frac{y_{++}}{4}\right)-f\left(\frac{y_{+-}}{4}\right)\right\}$ for each contributing nonlocal term. 


\section{Acknowledgements}

We are grateful to A. Linde for illuminating correspondence. This work was partially supported by NSF grant PHY-0653085 and by the Institute for Fundamental Theory at the University of Florida.

\section{References}

[1] R. P. Woodard, "Quantum Effects during Inflation," in Quantum Field Theory under the Influence of External Conditions (Rinton Press, Princeton, 2004) ed. K. A. Milton, pp. 325-330, astro-ph/0310757.

[2] V. F. Mukhanov and G. V. Chibisov, JETP Lett. 33 (1981) 532.

[3] A. A. Starobinskiı̌, JETP Lett. 30 (1979) 682.

[4] S. Weinberg, Phys. Rev. D72 (2005) 043514, hep-th/0506236; Phys. Rev. D74 (2006) 023508, hep-th/0605244.

[5] A. Bilandzic and T. Prokopec, "Quantum radiative corrections to slowwroll inflation", arXiv:0704.1905.

[6] D. Seery, "One-loop corrections to a scalar field during inflation", arXiv: 0707.3377; "One-loop corrections to the curvature perturbation from inflation", arXiv: 0707.3378.

[7] M. van der Meulen and J. Smit, "Classical approximation to quantum cosmological correlations", arXiv:0707.0842.

[8] M. S. Sloth, Nucl. Phys. B775 (2007) 78, hep-th/0612138.

[9] V. K. Onemli and R. P. Woodard, Class. Quant. Grav. 19 (2002) 4607, gr-qc/0204065; Phys. Rev. D70 (2004) 107301, gr-qc/0406098.

[10] T. Brunier, V. K. Onemli and R. P. Woodard, Class. Quant. Grav. 22 (2005) 59, gr-qc/0408080.

[11] E. O Kahya and V. K. Onemli, Phys. Rev. D76 (2007) 043512, gr-qc/0612026.

[12] T. Prokopec and R. P. Woodard, JHEP 0310 (2003) 059, astro-ph/0309593. 
[13] B. Garbrecht and T. Prokopec, Phys. Rev. D73 (2006) 064036, gr-qc/0602011.

[14] L. D. Duffy and R. P. Woodard, Phys. Rev. D72 (2005) 024023, hep-ph/0505156.

[15] S. P. Miao and R. P. Woodard, Phys. Rev. D74 (2006) 044019, gr-qc/0602110.

[16] T. Prokopec, O. Tornkvist and R. P. Woodard, Phys. Rev. Lett. 89 (2002) 101301, astro-ph/0205331; Ann. Phys. 303 (2003) 251, gr-qc/0205130.

[17] T. Prokopec and R. P. Woodard, Ann. Phys. 312 (2004) 1, gr-qc/0310056;

[18] E. O. Kahya and R. P. Woodard, Phys. Rev. D72 (2005) 104001, gr-qc/0508015 Phys. Rev. D74 (2006) 084012, gr-qc/0608049.

[19] T. Prokopec, N. C. Tsamis and R. P. Woodard, Class. Quant. Grav. 24 (2007) 201, gr-qc/0607094.

[20] T. Prokopec, N. C. Tsamis and R. P. Woodard, "Two Loop StressEnergy Tensor for Inflationary Scalar Electrodynamics," in preparation.

[21] N. C. Tsamis and R. P. Woodard, Phys. Rev. D54 (1996) 2621, hep-ph/9602317.

[22] L. H. Ford, Phys. Rev. D31 (1985) 710.

[23] F. Finelli, G. Marozzi, G. P. Vacca and G. Venturi, Phys. Rev. D71 (2005) 023522, gr-qc/0407101.

[24] N. C. Tsamis and R. P. Woodard, Ann. Phys. 321 (2006) 875, gr-qc/0506056.

[25] N. C. Tsamis and R. P. Woodard, Nucl. Phys. B474 (1996) 235, hep-ph/9602315; Ann. Phys. 253 (1997) 1, hep-ph/9602316.

[26] S. P. Miao and R. P. Woodard, Class. Quant. Grav. 23 (2006) 1721, gr-qc/0511140 Phys. Rev. D74 (2006) 024021, gr-qc/0603135. 
[27] A. A. Starobinskii, "Stochastic de Sitter (inflationary) stage in the early universe," in Field Theory, Quantum Gravity and Strings, ed. H. J. de Vega and N. Sanchez (Springer-Verlag, Berlin, 1986) pp. 107-126.

[28] R. P. Woodard, Nucl. Phys. Proc. Suppl. 148 (2005), 108 astro-ph/0502556.

[29] N. C. Tsamis and R. P. Woodard, Nucl. Phys. B724 (2005) 295, gr-qc/0505115.

[30] A. A. Starobinskiur and J. Yokoyama, Phys. Rev. D50 (1994) 6357, astro-ph/9407016.

[31] T. Prokopec, N. C. Tsamis and R. P. Woodard, Annals of Physics (in press), arXiv:0707.0847.

[32] V. F. Mukhanov, H. A. Feldman and R. H. Brandenberger, Phys. Rept. 215 (1992) 203.

[33] A. R. Liddle and D. H. Lyth, Phys. Rept. 231 (1993) 1, astro-ph/9303019.

[34] L. Smolin, Phys. Lett. 93B (1980) 95.

[35] A. D. Linde, Particle Physics and Inflationary Cosmology (Harwood, Chur, Switzerland, 1990), hep-th/0503202 "Inflationary Comsology," arXiv: 0705.0164 .

[36] E. O. Kahya and R. P. Woodard, "Quantum Gravity Corrections to the One Loop Scalar Self-Mass during Inflation," arXiv:0709.0536.

[37] N. C. Tsamis and R. P. Woodard, Commun. Math. Phys. 162 (1994) 217.

[38] R. P. Woodard, "de Sitter Breaking in Field Theory," in Deserfest: A Celebration of the Life and Works of Stanley Deser (World Scientific, Hackensack, 2006) eds. J. T. Liu, M. J. Duff, K. S. Stelle and R. P. Woodard, pp. 339-351.

[39] J. Schwinger, J. Math. Phys. 2 (1961) 407.

[40] K. T. Mahanthappa, Phys. Rev. 126 (1962) 329. 
[41] P. M. Bakshi and K. T. Mahanthappa, J. Math. Phys. 4 (1963) 1; J. Math. Phys. 4 (1963) 12.

[42] L. V. Keldysh, Sov. Phys. JETP 20 (1965) 1018.

[43] R. D. Jordan, Phys. Rev. D33 (1986) 444.

[44] K. C. Chou, Z. B. Su, B. L. Hao and L. Yu, Phys. Rept. 118 (1985) 1.

[45] E. Calzetta and B. L. Hu, Phys. Rev. D35 (1987) 495.

[46] L. H. Ford and R. P. Woodard, Class. Quant. Grav. 22 (2005) 1637, gr-qc/0411003. 